\title{
End-to-End Proportional Loss Differentiation in OBS Networks ${ }^{\star}$
}

\author{
Miguel A. González-Ortega, José C. López-Ardao, Pablo Argibay-Losada, \\ Andrés Suárez-González, Cándido López-García, Manuel Fernández-Veiga, \\ and Raúl F. Rodríguez-Rubio
}

Departmento Enxeñería Telemática, University of Vigo (Spain)

jardao@det.uvigo.es

\begin{abstract}
Proportional loss differentiation in OBS networks has recently received much attention since it allows to provide a quantitative differentiation between classes, so facilitating network operations and pricing to providers, but without the complexity involved by the absolute loss differentiation.

Although edge-to-edge differentiation must be the ultimate aim, most of the methods in the literature are per-hop-based, and it is well-known that guaranteeing per-hop proportional loss does not guarantee end-toend proportional loss.

In this paper we modestly try to fill the gap which exists in this field, and so, we propose and analyze a new method to obtain edgeto-edge proportional loss differentiation for WDM-based OBS networks. This method is based on the idea of trunk and wavelength reservation, already used in circuit switched networks and deflection routing in OBS networks.

Through extensive simulation experiments and analysis, we compare the performance of our mechanism to another mechanism proposed in the literature, based on using additional offset.
\end{abstract}

\section{Introduction}

The rapid advances in optical transmission technology have led to a wide deployment of WDM-based networks. In order to efficiently use the bandwidth in the optical domain, Optical Burst Switching (OBS) is considered as the most promising switching technology [1].

At the edge of OBS networks, incoming packets with the same destination and characteristics (class, quality of service, etc.) are assembled into big data packets, or data bursts. Before sending each burst, a control packet (Burst Header Packet - BHP) is sent over a signalling channel, and electronically processed in each intermediate node in order to reserve the necessary resources inside the core nodes. After an enough delay, the burst is sent, traversing the all-optical

\footnotetext{
* This work was supported by the "Ministerio de Educación y Ciencia" through the project TSI2006-12507-C03-02 of the "Plan Nacional de I+D +I" (partially financed with FEDER funds) and through the grant BES-2007-15562.
} 
path set by the prior reservation. However, given that the control packets are not acknowledged, a burst may be discarded owing to contention with other bursts at the bufferless optical switches.

Quality of Service (QoS) differentiation is still an open research issue in OBS networks. Since transfer delay in OBS is primarily determined by propagation delay, and bandwidth is implicitly provided by supporting loss guarantee, the focus of QoS support in OBS networks is to provide loss differentiation.

Different loss differentiation methods have been proposed in the literature. According to these, differentiation can be absolute or relative. Absolute methods allow to guarantee quantitative requirements (like an upper bound on loss probability for each class) but they need efficient admission control and resource provisioning mechanisms. On the other hand, in the relative methods the QoS of one class is defined relatively in comparison to other classes, providing differentiation only in a qualitative manner. Instead, they are quite simpler and cheaper.

If we analyze the methods proposed for loss differentiation (both absolute and relative) in the literature, we can see that they essentially use a few basic differentiation mechanisms, each one with its advantages and drawbacks: additional offset for the priority bursts ([2, [3]), intentional dropping of the less priority bursts $([4],[5], 6])$, priority-based preemption $([7])$, segmentation $([8],[9])$, classbased bandwidth assignment $([5,6])$, or reordering of burst scheduling at the core nodes (see, for example, [10]).

An special case of relative methods that have recently received much attention are those based on proportional QoS (see, for example, [4], [9] or [3]), since they allow to provide a quantitative differentiation between classes, so facilitating network operations and pricing to providers. The cost of achieving this quantitative differentiation, and not only qualitative, is a slightly higher complexity.

Although the use of absolute or proportional QoS is usually a compromise between the level of the QoS guarantees and the complexity (or cost), it seems obvious that edge-to-edge differentiation must be the ultimate aim. However, this aim is very difficult to achieve, and so, most of the above methods are perhop-based. But, it is well-known that guaranteeing per-hop proportional loss does not guarantee end-to-end proportional loss [11.

Some works $([5])$, however, go beyond trying to get close to a target end-to-end QoS using per-hop-based differentiation.

To the best of our knowledge, only the recent work in [3] proposes a edge-toedge proportional loss differentiation mechanism, referred to as FOTS (Feedbackbased Offset Time Selection). This mechanism is based on the use of an additional offset, beyond the needed time for burst scheduling, that will be adaptively selected according to end-to-end measurements of the Burst Loss Probability (BLP, from now on) for each flow. Nevertheless, as all the additional offset based mechanisms for loss differentiation, FOTS only works when JET (Just Enough Time) [1] is used as resource reservation mechanism, but not with JIT (Just In Time) [12], also widely used in the literature. 
In this paper we modestly try to fill the gap which exists in this field, and so, we propose a new edge-to-edge proportional loss differentiation mechanism for WDM-based OBS networks.

Our mechanism is based on a new basic differentiation mechanism, although it is inspired by the idea of trunk reservation in circuit switched networks [13] and wavelength reservation for deflection routing in OBS networks [14. We will refer to our mechanism as $A D E W A R$ (ADaptive End-to-end WAvelength Reservation).

We must note that $A D E W A R$ works with both JET and JIT reservation mechanisms. Moreover, $A D E W A R$ enjoys an interesting advantage since the Poisson traffic assumption allows us to calculate an analytical solution, based on the Erlang loss formula.

In any case, we have also used other more complex traffic models for, through extensive simulation experiments, studying ADEWAR and comparing it to FOTS when JET is used. End-to-end proportional loss differentiation is achieved in both cases, but some differences in performance were found.

The rest of the paper is organized as follows. Section 2 describes our mechanism $A D E W A R$. In Section 3 we show the simulation results and compare it to FOTS. The conclusions drawn are summarized in Section 4

\section{The $A D E W A R$ Mechanism}

In $A D E W A R$ we use the same idea of trunk reservation in circuit switched networks 13 and wavelength reservation for deflection routing in OBS networks 14, but now we apply this idea for loss differentiation in OBS.

So, we reserve a number of wavelengths on each link for the exclusive use of certain top-priority classes. That is, a burst of the Class of Service (CoS) $i$ will be scheduled for a link if the number of free wavelengths in this link is higher than $N-K_{i}$, and dropped otherwise. $N$ is the total number of wavelengths in that link.

In this paper we will refer to a flow as the burst traffic between a node-pair belonging to the same CoS. So, let $Q_{X-Y}^{i}$ be the QoS parameter for the flow corresponding to the $\operatorname{CoS} i$ between the node pair $X-Y$. Its value, ranging from 0 to 1 , is directly proportional to the number of allowed wavelengths to be used by the flow, that is, $K_{X-Y}^{i}=N \cdot Q_{X-Y}^{i}$.

If $Q=1$ for the highest priority $\operatorname{CoS}, K_{X-Y}^{1}=N$, that is, top-priority bursts will be always scheduled if there are free wavelengths. The value of $Q$ for the other classes between the nodes $X$ and $Y$ will be adjusted according to its target BLP, $B L P_{X-Y_{T A R}^{i}}$, that will be proportional to the BLP measured for the highest (or the lowest) priority CoS.

It is clear that such a technique can be used with both JET and JIT reservation mechanisms.

Due to the significant impact that an additional wavelength has on BLP, in case of $K_{X-Y}^{i}$ results in a non-integer number, one additional wavelength will be randomly allowed, proportionally to the fractional part. 
We propose two adaptive adjustment methods: one based on feedback and end-to-end measurements, and another one where each node adaptively makes adjustments based on local measurements. We will refer to the first one as endto-end adjustment, and to the second one as local adjustment.

In end-to-end adjustment, the adjustment and setting of the QoS parameter $Q$ is made at the source node, according to end-to-end measurements of the BLP, and this value will be used in all the nodes traversed by the burst. Specifically, all the bursts belonging to the flow between the node-pair $X-Y$ and $\operatorname{CoS} i$ will carry the same value $Q_{X-Y}^{i}$. It is obvious that the core nodes need to know the value of $Q$ for each burst. We propose that this value, set by the source node, travel into the corresponding control packet.

For the measurements of BLP, we propose the source nodes send "collector packets" to the destination nodes. These special packets will be replied indicating the number of bursts received at destination since the last "collector packet". This simple method can have drawbacks related to the convergence speed, but it is expected that traffic also changes slowly at the OBS networks, mainly used in the backbone.

With local adjustment, in order to avoid this potential problem, a sequence number is included in the control packets to allow each traversed node to calculate the number of lost bursts from the source. So, making the corresponding adjustment at each node, the end-to-end BLP converges rapidly to its target value on a per-hop basis.

In both cases, the adjustment consists in increasing $Q$ (at the source node, or at each intermediate node) when the last measured value of BLP is higher than the target, or decreased otherwise, so making the measured BLP gets close to its target value.

It seems clear that the per-hop adjustment will probably lead to a faster convergence and a better dynamic behaviour than the end-to-end adjustment; however, it is only valid when the flow travels along the same path from source to destination, that is, without using deflection or multipath.

The dynamics related to increasing and decreasing $Q$ are out of the scope of this paper and will be studied in a future work.

\section{Performance Evaluation}

We have studied the performance of $A D E W A R$ in comparison to FOTS through analysis and simulation experiments, also using the NSFNET network as in [3]. All the links have 50 wavelengths of $10 \mathrm{Gbps}$. and we assume full wavelengthconversion capability in every core node. Although $A D E W A R$ can be also used with JIT, we will use only JET as resource reservation mechanism, in order to be able to employ FOTS. LAUC (Last Available Unscheduled Channel) [15] was the scheduling algorithm used. Except for some particular experiments, the mean burst size is $320 \mathrm{Kbits}$, and the processing time for the control packets at the core nodes is fixed and equal to $4 \mu \mathrm{sec}$. For computational cost reasons, 
only a subset of source/destination node-pairs has been used, all of them with the same traffic pattern.

We consider three CoS and the following target BLPs: $B L P_{T A R}^{2}=5 \cdot B L P_{T A R}^{1}$ and $B L P_{T A R}^{3}=25 \cdot B L P_{T A R}^{1}$. In principle, each burst is randomly assigned to a $\operatorname{CoS}$ with probabilities $1 / 13$ for the $\operatorname{CoS} 1,3 / 13$ for the $\operatorname{CoS} 2$ and $9 / 13$ for the CoS 3. We will also consider other target BLPs and traffic ratios between the CoS.

In this scenario, we have conducted exhaustive simulation experiments to analyze the influence of many different factors on the performance of the mechanisms under study, mainly those related to the traffic pattern, both marginal distributions and correlation structure for burst interarrival times and burst sizes. Specifically, we will focus on:

- Correlation structure: By means of using a $\mathrm{M} / \mathrm{G} / \infty$ traffic generator [16], we model different correlation structures by means of only two parameters: the Hurst parameter $(\mathrm{H})$ for the LRD, and the one-lag autocorrelation coefficient $(R 1)$ for the SRD.

- Marginal distribution: We generate background $\mathrm{M} / \mathrm{G} / \infty$ processes to capture the desired correlation structure, but by means of a transformation that preserve the correlation structure, we change its marginal distribution (Exponential, Lognormal and Uniform), using different mean values (giving rise to different average traffic intensities) and variation coefficients $(\mathrm{VC}=$ standard deviation/mean).

As overall conclusions, we have observed that both ADEWAR and FOTS are able to provide end-to-end proportional loss differentiation (they have achieved the target proportional differentiation in all experiments we have carried out) and that FOTS suffers a slightly lower BLP than ADEWAR. However, this is achieved at the expense of increasing significantly the delay, due to the additional offset needed for differentiation, even prohibitive for some real-time applications. From now on, we will refer to this additional offset as FOTS offset.

We must also note that, although we have used local adjustment for $A D E$ $W A R$, the performance has been very similar for end-to-end adjustment.

In order to observe the performance degradation typically associated to differentiation, we have also studied the case with no differentiation (No Diff).

First, we will analyze in detail the simulation results about the global BLP, and then we will study the magnitude of the additional offset needed for differentiation in FOTS.

\subsection{Impact on Burst Lost Probability}

Figure 1 shows the global BLP obtained as a function of the mean traffic intensity when the burst arrival process for each flow is Poissonian, and the burst length follows an exponential distribution. From the simulation results, we can see that the BLP obtained by FOTS and No Diff are very similar for all loads, and slightly lower than those of $A D E W A R$. In Figure 1 (right), the corresponding fitting curves are also represented, showing that the BLP increases almost linearly with the traffic load for all the cases. 

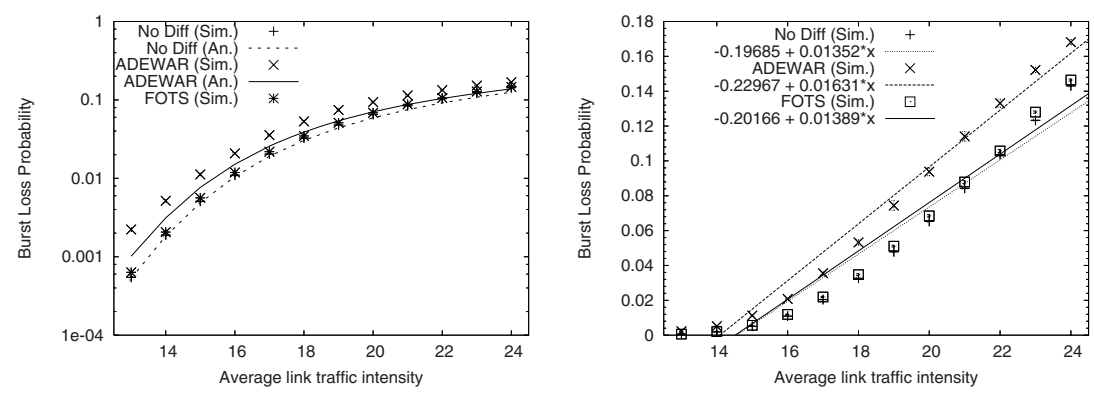

Fig. 1. Global Mean BLP for different traffic intensity values obtained by simulation, together with the analytical approximations (left) and the fitting curves (right)

Figure 1 (left) also shows the analytical results for $A D E W A R$ and No Diff. Since we use Poisson traffic patterns, BLP can be analytically estimated using the Erlang loss formulas, as in 13 and [17. We have also used a fixed-point iterative procedure, calculating the values of $Q_{X-Y}^{i}$, for each node-pair $X-Y$ and $\operatorname{CoS} i$, that allows us to achieve the target values of BLP, $B L P_{X-Y T A R}^{i}$, proportional to the BLP obtained for the top-priority CoS. We can see that the analytical results are slightly better than those of simulation. The reason is that the analytical model is optimistic, since it does not take into account the offset time between data bursts and BHPs.

We have also studied other parameters like the differentiation degree requirements, the BHP processing time at the core nodes and the ratio of traffic for each CoS. Although the results obtained were not included for extension reasons, we will make some comments.

We have found that BLP increases slightly with BHP processing time in all cases. The reason is that the minimum offset time between the BHP and the data bursts also increases with it. So, on the one hand, data bursts arrive at the core nodes with very different offset times, and this makes difficult to provide good resource provisioning. On the other hand, LAUC scheduling algorithm does not make use of the free wavelength gaps before the scheduled bursts, due to the offset time and, obviously, such wasted gaps will be larger when offset time increases.

However, we could observe that the differentiation requirements hardly affect the global BLP obtained by FOTS, although there was a slight increase in the BLP of $A D E W A R$ when the target differentiation was very stringent.

In the same way, the ratio of traffic for each $\mathrm{CoS}$ does not have a big impact on global BLP.

Impact of the Burst Interarrival Time. With respect to the marginal distribution, the performance changes in a similar way for the three cases, keeping the relative differences between them unchanged (see Figure 2 (left)). However, we can note that the lighter the tail of the marginal distribution is, the greater BLP is, since the smallest values of the interarrival time distribution are the 

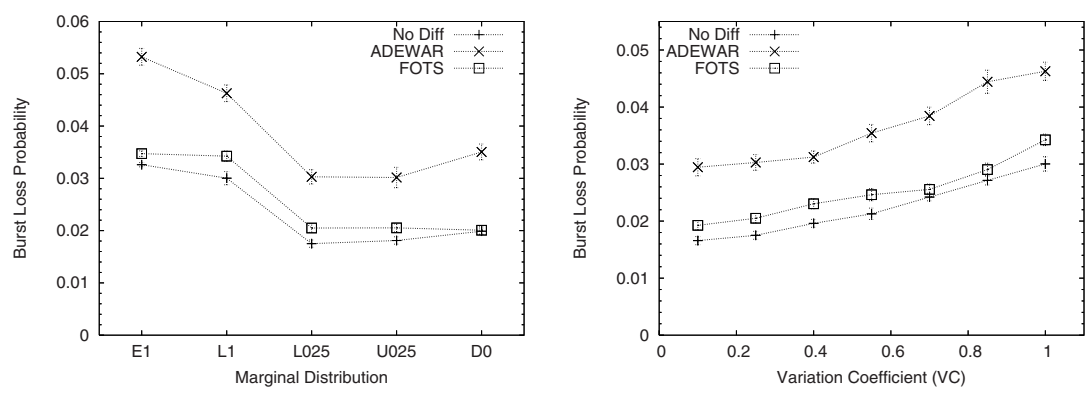

Fig. 2. Global Mean BLP for various marginal distributions: Exponential (E1), Lognormal with $\mathrm{VC}=1$ (L1) and $\mathrm{VC}=0.25$ (L025), Uniform with $\mathrm{VC}=0.25$ (U025), and deterministic (D0) (left) and as a function of VC with Lognormal distribution (right) of the burst interarrival time
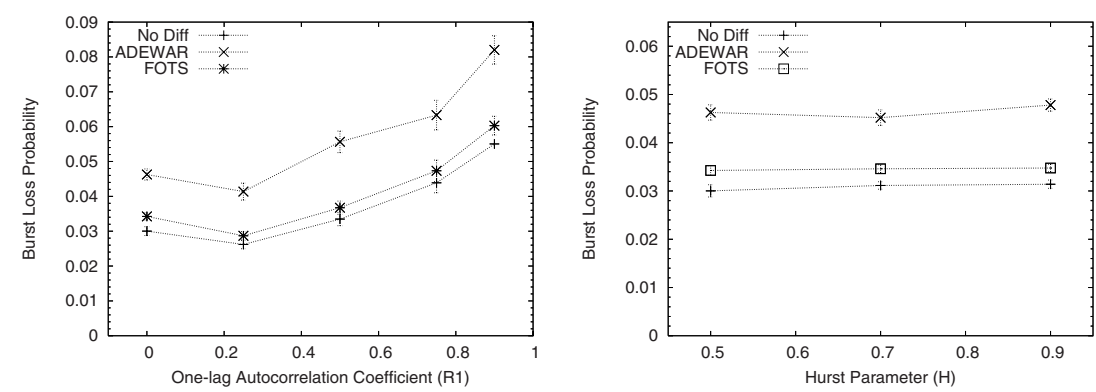

Fig. 3. Global Mean BLP depending on the SRD, represented by $R 1$ (left) and on the LRD, represented by $H$ (right) of the burst interarrival time. L1 marginal distribution was used.

most probable to occur, and so overlapping is also more probable. For this reason, the Exponential gives rise to greater values of BLP than the Lognormal. On the other hand, BLP increases with the value of VC (see Figure 2 (right)), but for small values the difference is not significant (for example, BLPs obtained for D0 and for L025 are similar)

In Figure 3 we show the effects of the correlation structure, both SRD and LRD. Again, the influence of $R 1$ is similar for all the cases. The increase of $\mathrm{VC}$ or R1 always degrades the network performance, because they cause traffic burstiness. This degradation is more significant for the case of R1. However, we can observe that the effects of LRD of the burst interarrival time is negligible. This can be explained by the lack of buffers in the optical paths, that introduces a reset effect in the correlations.

Impact of the Burst Sizes. We have also studied the impact of several characteristics of the burst size in the global BLP, using Poisson arrivals.

We can see that BLP decreases very slightly with the mean burst size, and this decrease is more significant for $A D E W A R$ (see Figure 4 (left)). That is 

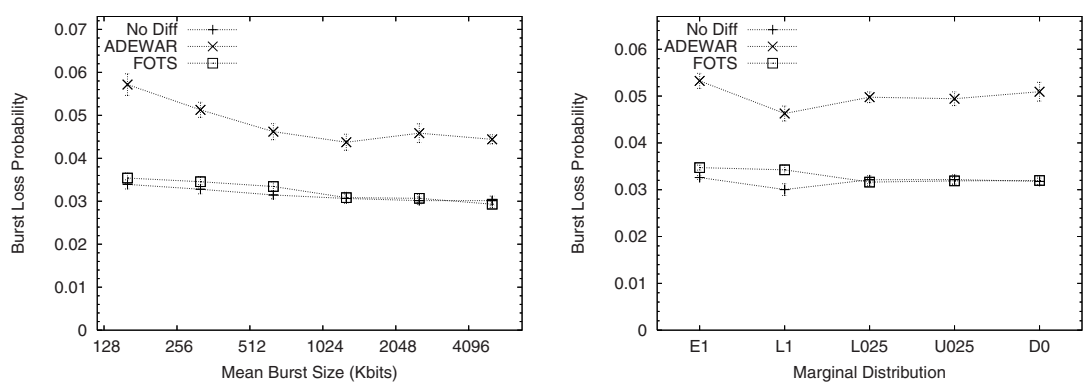

Fig. 4. Global Mean BLP as a function of the mean value (left) and the marginal distribution (right) of the burst size. L1 marginal distribution was used.
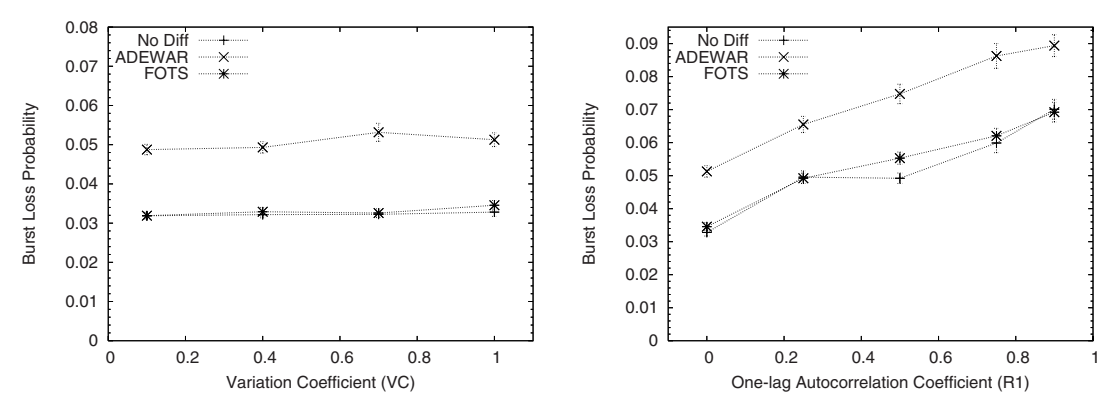

Fig. 5. Global Mean BLP depending on the VC (left) and on the SRD (right) of the burst size. L1 marginal distribution was used.

because for a given load the effect of increasing burst size is similar to that of decreasing the offset time. On the the one hand, as we reduce the number of bursts sent per time unit, the impact of the disparity between the order of the burst arrivals and the resource reservations is also reduced. So, it is easier to provide a good resource provisioning. On the other hand, wasted gaps caused by LAUC algorithm become relatively less significant.

With respect to the marginal distribution of the burst size and its VC (see Figures 4 (right) and 5 (left)), we can assert that the influence of both parameters on BLP is negligible.

Regarding the correlation structure, we can see that the effect of SRD, as happened with the burst interarrival time, is important since traffic is more burstiness. So, BLP increases considerably and the impact is very similar for the three cases. However, we have observed that, again as in the case of burst interarrival time, LRD has hardly impact on BLP.

\subsection{Impact on FOTS Offset}

In this section, we focus our analysis on the magnitude of the FOTS offset, considering the same scenarios as those of the previous section. Burst sizes follow 

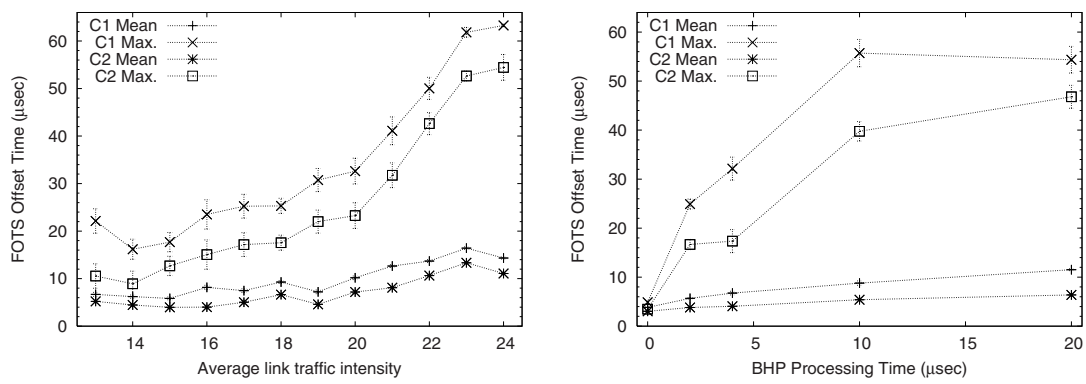

Fig. 6. FOTS offset as a function of the traffic intensity (left) and of the BHP processing time (right)

an exponential distribution, and burst arrivals are Poissonian. The average number of hops per path was 2.465, and the BHP processing time at the core nodes was $4 \mu \mathrm{sec}$. So, the mean minimum offset time, or basic offset, results $2.465 \cdot 4 \mu$ sec. $=9.86 \mu$ sec.

Our objective is to discuss whether the FOTS offset is significant, both in absolute terms and compared with the basic offset, and so whether FOTS could be inadequate for some real-time applications.

The figures show the average FOTS offset (Mean) and the maximum between all the flows (Max.) for $\operatorname{CoS} 1$ and 2 ( $C 1$ and $C 2)$.

Figure 6 (left) shows the effect of the traffic intensity. We can observe that as BLP increases with the load, the FOTS offset also increases. A simple case can explain this. Let us consider one single OBS link with $N$ wavelengths, Poissonian burst arrivals, and two priority classes $(A>B)$, with traffic intensities $I_{A}$ and $I_{B}$. In this case we define the isolation degree achieved by FOTS, $\alpha$, as the fraction of class $B$ traffic that does not compete for the resources with class $A$ bursts. Obviously, $\alpha$ increases with FOTS offset. If we reasonably suppose that global BLP hardly depends on the FOTS offset, then, by applying the Erlang-B loss formula $\left(E_{N}(I)\right)$, we obtain that $I_{A} B L P_{A}+I_{B} B L P_{B}=\left(I_{A}+I_{B}\right) E_{N}\left(I_{A}+I_{B}\right)$. Moreover we have $B L P_{A}=E_{N}\left(I_{A}+(1-\alpha) I_{B}\right)$, and $B L P_{B}=\left(\left(I_{A}+I_{B}\right) E_{N}\left(I_{A}+\right.\right.$ $\left.\left.I_{B}\right)-I_{A} B L P_{A}\right) / I_{B}$. It is easy to see from the above equations, that the quotient $B L P_{B} / B L P_{A}$ decreases with traffic intensity and increases with $\alpha$, provided that $I_{A}+I_{B}$ and the rest of the parameters remain fixed. Therefore, the higher the traffic load is, the larger the FOTS offset must be, in order to increase $\alpha$ and hold a given loss differentiation.

We can also see in Figure [6] (left) that for both C1 and C2 FOTS offset is quite large. So, the average values are comparable to the basic offset $(9.86$ $\mu$ sec.), that is, the transmission delay due to OBS offset time has doubled on average. But, if we focus on the maximum values, they are even much larger, peaking at over $60 \mu \mathrm{sec}$. for the highest loads. Therefore, for some flows, FOTS has increased offset time by more than 7 times, and this additional delay could be too long for real-time applications.

In Figure 6 (right) we can observe that FOTS offset increases with the BHP processing time. As already said, the reason is that the basic offset also increases 



Fig. 7. FOTS offset depending on the marginal distribution (left) and on the SRD (right) of the burst interarrival time

with it, and so a larger FOTS offset is needed to achieve a certain isolation degree $(\alpha)$. Moreover, the results showed that, as expected, FOTS offset was larger when differentiation was more demanding, since $\alpha$ had to be greater, too.

We could also observe, as pointed out in [3], that the FOTS offset increases with the ratio of the top-priority traffic.

As a conclusion, we find again that the mean FOTS offset is comparable to the basic offset, but for certain flows it can become even several times larger.

In Figure 7 we can see again that, as a general rule, the conditions which make BLP increase also make FOTS offset increase since, using the same isolation degree among CoS, the proportional BLP differentiation decreases when the global BLP increases, as it was already said. So, the FOTS offset increases with the SRD degree and the value of $\mathrm{VC}$, and with the use of light-tailed marginal distributions (like Exponential) of the burst interarrival time.

We have also studied the influence of the VC and the LRD of the burst interarrival time on the FOTS offset. As expected, we have found that both parameters have a similar impact on the global BLP and on the FOTS offset. So, FOTS offset slightly increases with VC, but hardly depends on LRD.

Figure 8 shows the FOTS offset obtained by varying the mean value and the $R 1$ parameter of the burst sizes.

We can note that the FOTS offset notably increases with the burst size. This can be explained with a simple example. Let us assume null basic offset and bursts of constant length $L$. In this case, a burst whose arrival time at a core node is $t$ could be blocked only by bursts that arrive into the collision interval $[t-L, t]$. It is easy to see that, for two priority levels, obtaining a certain isolation degree $\alpha$ between them implies to change the collision interval of the highest priority to $[t-L, t-\alpha L]$. Therefore, the FOTS offset must be $L \alpha$, and so it increases with the burst size. Similar explanations can be applied to more complex scenarios.

The collision interval depends on the maximum burst size. So, FOTS offset increases when large values of VC and heavy-tailed distributions are used for the burst sizes. Likewise, and due to the increase of the global BLP, the SRD also contributes to increase the FOTS offset (see Figure 8 (right)). 

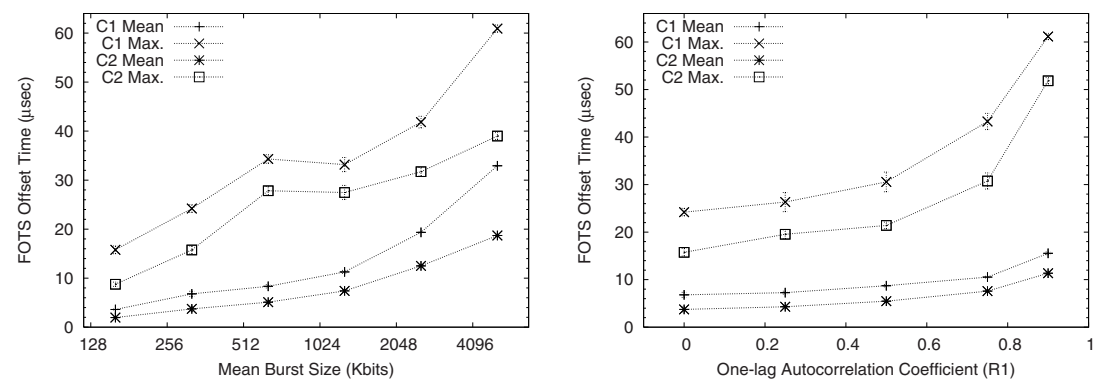

Fig. 8. FOTS offset depending on the mean burst size (left) and on the one-lag autocorrelation coefficient of the burst sizes (right)

We have also analyzed the effect of the LRD of the burst sizes, but the results again reveal a negligible influence on the FOTS offset, basically for the same reasons, that is, the reset effect imposed by the lack of buffers in OBS networks.

\section{Conclusions}

In this paper we propose and analyze a new technique to obtain edge-to-edge proportional differentiation for WDM-based OBS networks. We call this method $A D E W A R$ (ADaptive End-to-end WAvelength Reservation). ADEWAR is based on the wavelength reservation, previously used in circuit switched networks and deflection routing in OBS networks.

Through analysis and extensive simulation experiments, we compare the performance of our mechanism to FOTS (Feedback-based Offset Time Selection), another feedback-based mechanism proposed in the literature that uses additional offset for achieve differentiation. This mechanism has some disadvantages compared to $A D E W A R$, since it only works with the JET reservation mechanism and it uses an end-to-end feedback-based adjustment, that converges slower than our local adjustment.

Both ADEWAR and FOTS achieve end-to-end proportional loss differentiation, and although FOTS causes slightly less burst losses than our technique. the additional delay required for differentiation could be too long for real-time applications, specially if data bursts are large and with high traffic loads.

\section{References}

1. Qiao, C., Yoo, M.: Optical burst switching (OBS)-a new paradigm for an optical internet. Journal of High Speed Networks 8(1), 69-84 (1999)

2. Yoo, M., Qiao, C., Dixit, S.: QoS performance of optical burst switching in IPover-WDM networks. IEEE Journal on Selected Areas in Communications 18(10), 2062-2071 (2000) 
3. Tan, S.K., Mohan, G., Chua, K.C.: Feedback-based offset time selection for endto-end proportional QoS provisioning in WDM optical burst switching networks. Computer Communications 30(4), 904-921 (2007)

4. Chen, Y., Hamdi, M., Tsang, D.H.K.: Proportional QoS over OBS networks. In: Proc. of GLOBECOM, November 2001, vol. 3, pp. 1510-1514. IEEE, Los Alamitos (2001)

5. Zhang, Q., Vokkarane, V., Jue, J., Chen, B.: Absolute QoS differentiation in optical burst-switched networks. IEEE Journal on Selected Areas in Communications 22(9), 1781-1795 (2004)

6. Zhou, B., Bassiouni, M.A.: Supporting differentiated quality of service in optical burst switched networks. Optical Engineering 45(1) (January 2006)

7. Liao, W., Loi, C.-H.: Providing service differentiation for optical-burst-switched networks. Journal of Lightwave Technology 22(7), 1651-1660 (2004)

8. Vokkarane, V.M., Jue, J.P.: Prioritized burst segmentation and composite burstassembly techniques for QoS support in optical burst-switched networks. IEEE Journal on Selected Areas in Communications 21(7), 1198-1209 (2003)

9. Gurusamy, M., Tan, C., Lui, J.: Achieving proportional loss differentiation using probabilistic preemptive burst segmentation in optical burst switching WDM networks. In: Proc. of GLOBECOM, November-December 2004, vol. 3, pp. 1754-1758. IEEE, Los Alamitos (2004)

10. Yang, M., Zheng, S.Q., Verchere, D.: A QoS supporting scheduling algorithm for optical burst switching DWDM networks. In: Proc. of GLOBECOM, November 2001, vol. 1, pp. 86-91. IEEE, Los Alamitos (2001)

11. Chen, Y., Qiao, C., Hamdi, M., Tsang, D.H.K.: Proportional differentiation: a scalable QoS approach. IEEE Communications Magazine 41(6), 52-58 (2003)

12. Wei, J.Y., McFarland, R.I.: Just-in-time signaling for WDM optical burst switching networks. Journal of Lightwave Technology 18(12), 2019-2037 (2000)

13. Krupp, R.S.: Stabilization of alternate routing networks. In: Proc. of ICC, June 1982, pp. 1-31. IEEE, Los Alamitos (1982)

14. Zalesky, A., Vu, H., Rosberg, Z., Wong, E., Zukerman, M.: Modelling and performance evaluation of optical burst switched networks with deflection routing and wavelength reservation. In: Proc. of INFOCOM, March 2004, IEEE, Los Alamitos (2004)

15. Xiong, Y., Vandenhoute, M., Cankaya, H.C.: Design and analysis of optical burstswitched networks. In: Senior, J.M., Qiao, C., Dixit, S. (eds.) Proc. of SPIE, SPIE, vol. 3843, pp. 112-119 (1999)

16. Suárez-González, A., López-Ardao, J.C., López-García, C., Fernández-Veiga, M., Rodríguez-Rubio, R.F., Sousa-Vieira, M.E.: A new heavy-tailed discrete distribution for LRD M/G/inf sample generation. Performance Evaluation 47(2-3), 197 $219(2002)$

17. Argibay-Losada, P., Suárez-González, A., Veiga, M.F., Rodríguez-Rubio, R., López-García, C.: Evaluation of optical burst switching as a multiservice environment. In: Akyildiz, I.F., Sivakumar, R., Ekici, E., de Oliveira, J.C., McNair, J. (eds.) NETWORKING 2007. LNCS, vol. 4479, pp. 970-980. Springer, Heidelberg (2007) 